\title{
Patient Health Monitoring System and Prediction using Data Analytics
}

\author{
Padmashree T \\ Department of Information Science and Engineering \\ R.V.College of Engineering, Bengaluru \\ Dr.N.K.Cauvery \\ Department of Information Science and Engineering \\ R.V.College of Engineering, Bengaluru \\ Anirudh.V.C \\ Department of Information Science and Engineering \\ R.V.College of Engineering, Bengaluru \\ Punith Kumar \\ Department of Information Science and Engineering \\ R.V.College of Engineering, Bengaluru
}

\begin{abstract}
Every year more and more individuals are diagnosed with diseases which are chronic leading to rise in the requirement of enormous medical resources. With the healthcare centres being loaded with patients, importance is being given to remote monitoring techniques. Technology for healthcare industry is growing rapidly over the years. Data acquisition and monitoring systems for healthcare are estimated to have the largest growth in coming years. The proposed system aims at improving healthcare management by analysing health parameters like blood pressure, body temperature, heart rate and predicting heart problems. This is of great advantage especially when the patient is mobile. The system aims to analyse health parameters and alert the application user in case of any abnormalities. The patient can view his health data on his phone and the doctor can view the health information regarding all the patients that he is treating. Healthcare systems of late have more applications in the area of Cardiac diseases. This system which is specifically developed to predict heart problems of the application users who are patients previously diagnosed with heart disease. It provides digital information of the patients which is centrally stored. This data can be used for designing an improved healthcare delivery system. It continuously monitors health parameters like blood pressure (systolic and diastolic), body temperature and heart rate. These parameters are provided as input to the Weka library that is integrated to the android application. Multilayer perceptron algorithm in Weka is used to find the similarity in pattern between the trained data set which consists of health parameters of patients with heart problems and test data which contains the health data of the application users.
\end{abstract}

Keywords: Healthcare, Android, Analysis, Multilayer perceptron, Weka

\section{I.INTRODUCTION}

In the present day scenario, clinicians are relying extremely on technology to assist in patient monitoring. Also, healthcare systems designed with remote monitoring have been proven to improve patient's quality of life at reduced costs. Lately, it has been generally accepted that the benefits of remote monitoring overtake its drawbacks, and therefore $\mathrm{many}$ applications are being developed to address the needs of mobile health. With the emergence of IoT (Internet of Things), wearable devices have been developed which measures various health parameters like heart rate, body temperature etc. and display it on the mobile phone which can be used to monitor the health of a person.

The work carried out is a health data analysis system which can be used with smart phones and aims to analyse collected health parameters in the background and detect early signs of health problems and prevent further complications. The system also provides interaction between the doctor and the application users by providing access of the health data to the doctors. The work done concentrates more on software part of monitoring and analysis as hardware health data acquisition systems already exist. Data acquisition is simulated here using existing datasets which can be used for further analysis. 
The purpose of this work is to improve health monitoring using android application in mobile phones. The patient can view the history of his health data on his phone and the doctor can view the health information regarding all the patients who are being monitored by him. When the data is analysed and the patient's condition is critical the patient will be informed about the location of the nearest hospital around him using global positioning system (GPS) and a message regarding the patient's condition will be sent to the concerned doctor along with the hospital details he has reported to.

The scope of this work mainly lies in the health sector and to the people who need continuous monitoring of health data. The system can also bridge the gap between doctors and patients by allowing doctors to view the patient's data anytime they want to.

\section{LITERATURE SURVEY}

In the paper" Mobile Phone-Based Health Data Acquisition System Using Bluetooth Technology" by Majid A. Al-Taee, Nadine A. Jaradat, Dima M. Abu Ali, 2013 [1] addresses development of a mobile health (mHealth) system for remote acquisition of patient data using Bluetooth technology. It is accomplished by using Bluetooth to transfer the health data from the local medical device to the local storage. This data can be stored in the remote server whenever internet is available. The greatest advantage of this approach is that the patient could be mobile.

"Wireless data acquisition and monitoring for healthcare services systems" by Amir Hoshang Kioumars, 2014 [2] outlines the design and development of cost effective and reliable sensing device for collecting real time health vital signs such as heart beat and body temperature. AT micro controller and Zigbee technology is used for wireless communication that can be used to acquire patient's health data.

In, "A Proposal of a Mobile Health Data Collection and Reporting System for the Developing World" by Deo Shao, 2012 [3] researchers use data already available in the hospitals and provide the statistics of patient's health data. It also helps in keeping the patient medical history.

"Machine Learning with WEKA" by Svetlana S. Aksenova, 2004[4] provides a comprehensive tutorial to Weka tool for freshers. Various functionalities of the tool have been utilized as a part of this work.

In, "Predictive Data Mining for Medical Diagnosis: An Overview of Heart Disease Prediction" by Jyoti Soni, ujma Ansari, Dipesh Sharma, Sunita Soni, 2013[5], the researchers provide a survey of current techniques of knowledge discovery in databases using data mining techniques that are in use in today's medical research particularly in Heart Disease Prediction. They compare the performance of each data mining technique using the same dataset.

"Use WEKA in your Java code" by Binh Tran, 2013[6] gives an insight to the usage of Weka in the Java code. It provides various libraries, classes and methods that can be used for classification and data modelling.

"WEKA-Experiences with a Java Open-Source Project", by Remco R. Bouckaert, Eibe Frank, Mark A. Hall, Geoffrey Holmes, Bernhard Pfahringer, Peter Reutemann, Ian H.Witten, 2010 [7] provides the instructions to interface Weka with Java. Other than this, it also reviews the concepts of project management and decisions that can impact the development of application.

With smart phones becoming ubiquitous, it is easier to have applications that monitor patient's health continuously and analyse the same. Data acquisition systems already exist that acquires, stores and displays health data to the user. The proposed system makes use of this data for analysis and alert the user and concerned doctor. The system aims to prevent health complications by detecting the problems well ahead of complications.

\section{III.METHODOLOGY}

Initially, the doctors should register by providing their details along with their patient details. The patients also should register by providing their personal details. The health related data of each patient will be stored in the database at the server end and contain values obtained at real time. The patient and doctor will be able to view health related data which can be used to supervise patient's health. When analysis is performed on health data of the patient, in case of critical condition a notification will be sent to the concerned doctor. In case of critical condition, the location of the nearest hospital will be sent to the patient as well as the concerned doctor.

\section{A. System Architecture}

Mobile-Phone based Health Data Analysis system is decomposed into sub-systems that provide some related set of 


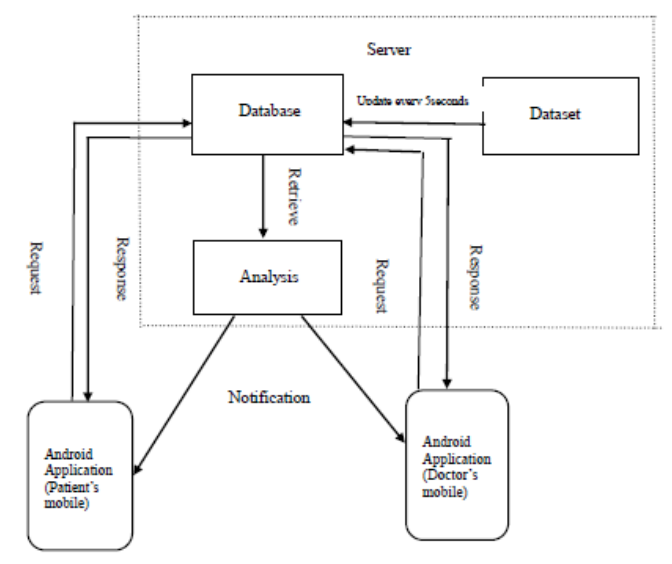

Figure 1 System Architecture

\section{IMPLEMENTATION}

In this system, Java, PHP and XML languages have been used which have been integrated with Android Studio Integrated Development Environment (IDE).

\section{B. Data analysis:}

Waikato Environment for Knowledge Analysis (Weka) is a popular suite of machine learning software written in Java, developed at the University of Waikato, New Zealand. It is free software licensed under the GNU General Public License.

Weka tool hosts numerous algorithms that can be used for data analysis. In this work, Multilayer perceptron (MLP) algorithm is used for analysis. A multilayer perceptron is a feed forward artificial neural network model that maps the input data onto appropriate output. An MLP constitutes multiple layers of nodes in the format of a directed graph, with each layer completely connected to the next one. Except for the input nodes, each node is a neuron (or processing element) with a nonlinear activation function. MLP utilizes a supervised learning technique called back propagation for training the network. MLP is a modification of the standard linear perceptron and can distinguish data that are not linearly separable.

\section{Task Implementation}

Login and Registration is accomplished by providing a unique id to registered doctors and patients. The details provided by them are stored in the MySQL database at the server.

The health data of the patient is simulated by retrieving the values stored in the database every minute and displaying it at the user interface. This is done using android volley library that sends JSON requests to server and parses the JSON response. These parameters obtained are given as input to the Weka library which contains a collection of algorithms and predictive models. The multilayer perceptron algorithm is used for analysis. The algorithm recognizes the pattern from the trained data which consists of the health data of patients during a heart attack and checks for the same pattern in the patient using the android application. In case the pattern of health data matches, an SMS alert is sent to the patient's consulting doctor. The patient is provided the locations of the nearby hospitals using Google Place API's and Google Maps API's

\section{V.RESULTS}

An user friendly GUI has been provided as a part of this application which is designed to have two views: one for the patient and other for the doctor. The UI dynamically updates to show the updated health parameters every minute.

The analysis module is invoked on receiving the health data

The health data is provided as the input to the multilayer perceptron algorithm. The patient's previous health data and the current data are compared against the training set containing data of the patients with heart problem and checks for similar patterns. In case of similar patterns the android application notifies the patient with locations of the nearby hospitals and also sends an SMS alert to the consulting doctor. 


\section{A. Inference}

The proposed system continuously monitors and analyses health parameters like Blood Pressure (BP), body temperature and heart rate using Multilayer perceptron that has an accuracy of $92 \%$ which is more than other classifiers like naïve Bayesian and decision trees. The system developed allows the patient to be mobile while his health parameters are being monitored. Patient information is made available to all healthcare providers through the android application.

B. Snapshots

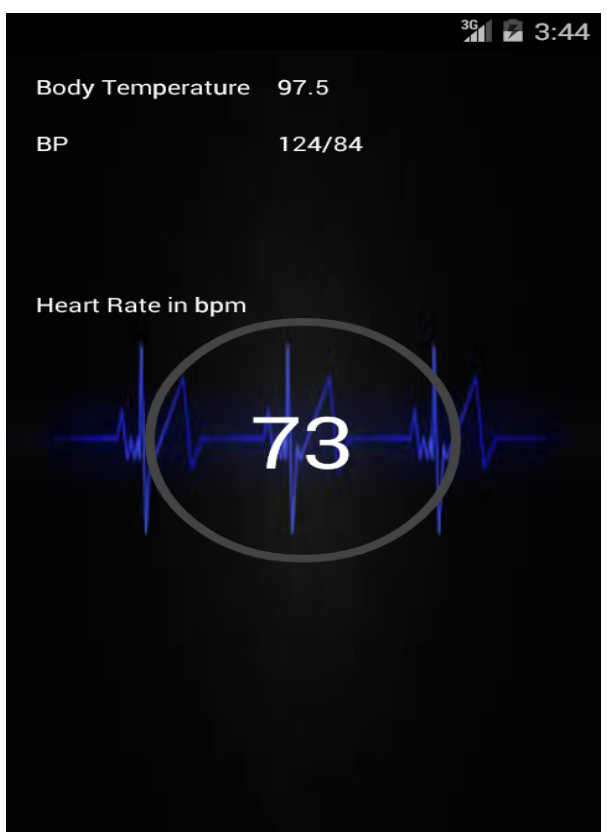

Figure 4 Data View Activity

Figure 2, shows the health parameters heart rate, body temperature and blood pressure of the patient which are retrieved from the server and displayed

Figure 3, shows the screenshot of the GPS module which detects the patient's current location and finds the nearby hospitals that he can visit
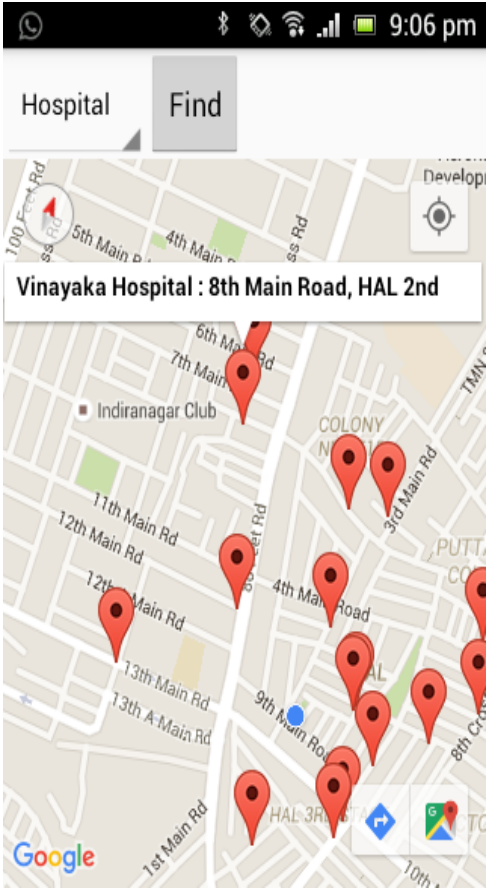
Figure 5 GPS module

Multiple techniques and methods exist to solve different issues involved in monitoring the health data and predicting heart problems each of which as its own pro's and con.

\section{LIMITATIONS AND FUTURE ENHANCEMENTS}

Currently, the system is designed for only specific set of patients who have heart related issues which can be further extended to monitor other kinds of diseases. The accuracy of prediction can be further increased. The data set on which the application is tested has around 1000 values for health parameters considered. This can be further expanded to test the accuracy of prediction.

\section{REFERENCES}

[1] Deo Shao, "A Proposal of a Mobile Health Data Collection and Reporting System for the Developing World," Malmö University, 2012, pp. 43-57

[2] Amir Hoshang Kioumars, "Wireless data acquisition and monitoring for healthcare services systems," Massey University, 2011, pp. 50-145

[3] Majid A. Al-Taee, Nadine A. Jaradat, Dima M. Abu Ali, "Mobile Phone-Based Health Data Acquisition System Using Bluetooth Technology," IEEE Jordan Conference, Amman, Dec. 2011, pp6-8

[4] Svetlana S. Aksenova, "Machine Learning with WEKA", California State University, 2004

[5] Jyoti Soni, ujma Ansari, Dipesh Sharma, Sunita Soni, "Predictive Data Mining for Medical Diagnosis: An Overview of Heart Disease Prediction", International Journal of Computer Applications, Vol. 17, No.8, March 2013

[6] Binh Tran, "Use WEKA in your Java code", 2013

[7] Remco R. Bouckaert, Eibe Frank, Mark A. Hall, Geoffrey Holmes, Bernhard Pfahringer, Peter Reutemann, Ian H.Witten, "WEKAExperiences with a Java Open-Source Project", Journal of Machine Learning Research 11 2533-2541, 2010

[8] G. Malkar, "Healthcare without bounds: trends in remote patient monitoring 2009," SpyGlass Consulting Group, March 2009.

[9] Ian H. Witten; Eibe Frank; Mark A. Hall (2011). "Data Mining: Practical machine learning tools and techniques, 3rd Edition". Morgan Kaufmann, San Francisco. Retrieved 2011-01-19.

[10] G. Holmes; A. Donkin; I.H. Witten (1994). "Weka: A machine learning workbench" (PDF). Proc Second Australia and New Zealand Conference on Intelligent Information Systems, Brisbane, Australia. Retrieved 2007-06-25.

[11] S.R. Garner; S.J. Cunningham; G. Holmes; C.G. Nevill-Manning; I.H. Witten (1995). "Applying a machine learning workbench: Experience with agricultural databases" (PDF). Proc Machine Learning in Practice Workshop, Machine Learning Conference, Tahoe City, CA, USA. pp. 14-21. Retrieved 2007-06-25.

[12] Rosenblatt, Frank. x. Principles of Neurodynamics: Perceptrons and the Theory of Brain Mechanisms. Spartan Books, Washington DC, 2001

[13] Rumelhart, David E., Geoffrey E. Hinton, and R. J. Williams. "Learning Internal Representations by Error Propagation". David E. Rumelhart, James L. McClelland, and the PDP research group. (editors), Parallel distributed processing: Explorations in the microstructure of cognition, Volume 1: Foundations. MIT Press, 2003.

[14] Cybenko, G. 1989. Approximation by superpositions of a sigmoidal function Mathematics of Control, Signals, and Systems, 2(4), $303-314$.

[15] Jayshril S. Sonawane, D. R. Patil "Prediction of heart disease using multilayer perceptron neural network ", Information Communication and Embedded Systems, International Conference, Chennai, Feb. 2014, pp. 1-6

[16] Syed Umar Amin, Kavita Agarwal, Rizwan Beg "Genetic neural network based data mining in prediction of heart disease using risk factors ", Information \& Communication Technologies, IEEE Conference, Jeju Island, Apr. 2013, pp. 1227 - 1231

[17] Feng Xiao, Yimin Chen, Ming Yuchi, Mingyue Ding "Heart Rate Prediction Model Based on Physical Activities Using Evolutionary Neural Network", Genetic and Evolutionary Computing, Fourth International Conference, Shenzhen, Dec. 2010 , pp. 198 - 201

[18] Hongmei Yana, Yingtao Jiangb, Jun Zhenge, Chenglin Pengc, Qinghui Lid "A multilayer perceptron-based medical decision support system for heart disease diagnosis”, Expert Systems with Applications, Vol. 30, no. 2, Feb. 2010, pp. 272-281

[19] Sellappan Palaniappan, Rafiah Awang "Intelligent heart disease prediction system using data mining techniques", IEEE/ACS International Conference on Computer Systems and Applications, Doha, Apr. 2008, pp. 108 - 115

[20] Anchana Khemphila, Veera Boonjing "Heart Disease Classification Using Neural Network and Feature Selection", Systems Engineering (ICSEng), 21st International Conference, Las Vegas, Aug. 2011, pp. 406 - 409 